\title{
Sex-related differences in acute and chronic pain: a bench to bedside perspective
}

\author{
Katherine A. Mifflin, BSc • Bradley J. Kerr, PhD
}

Received: 13 December 2012/ Accepted: 19 December 2012/Published online: 4 January 2013

(C) Canadian Anesthesiologists' Society 2013

Chronic pain affects the daily lives of millions. It has been estimated that nearly one-quarter of the world's population suffers from chronic pain, making it the most common human health problem. ${ }^{1}$ The most recent estimates of the prevalence of chronic pain in Canada indicate that approximately $29 \%$ of the population experiences chronic pain, with nearly $80 \%$ of these people reporting their pain as moderate to severe. ${ }^{2}$ Interestingly, there is increased pain sensitivity and a higher prevalence of chronic pain conditions in females than in males. ${ }^{3,4}$ To date, however, there has been little basic research examining these sex differences from a mechanistic standpoint, with researchers either doubting or simply ignoring the evidence. Nevertheless, numerous studies and a recent review of the literature provide convincing evidence that there are indeed sex differences relating to chronic and acute pain. ${ }^{4}$

Sex differences have been examined in many areas of biological research, particularly in the field of molecular neuroscience. Numerous studies have shown somatic and hormonal sex differences at a molecular level, and these

K. A. Mifflin, BSc · B. J. Kerr, PhD

Centre for Neuroscience, University of Alberta, Edmonton, AB, Canada

B. J. Kerr, $\mathrm{PhD}(\square)$

Department of Anesthesiology and Pain Medicine, University

of Alberta, Clinical Sciences Building, 8-120, Edmonton,

AB T6G 2G3, Canada

e-mail: bradley.kerr@ualberta.ca

B. J. Kerr, PhD

Department of Pharmacology, University of Alberta, Edmonton, $\mathrm{AB}$, Canada molecular differences between sexes are often reflected by differences in the outcome and progression of disease. This can been seen in Alzheimer's disease, depression, Huntington's disease, and cerebral ischemia, as well as in models of cognitive ability. ${ }^{5}$ Despite the well-described sex differences in pain and in other disease models, basic pain research has been slow to adopt systematic study of these differences. Common barriers to the study of sex differences in the basic mechanisms of pain include doubt for the need to study sex differences (an impact by conflicting studies devaluing the importance of sex studies), possible complications from different hormonal influences on behaviour, and the complexity of trying to determine the interacting roles of sex, pain, and the secondary factors associated with pain (e.g., depression).

Dr. Jeffery Mogil of McGill University addressed these concerns in a recent review of both clinical and basic studies examining sex differences. Dr. Mogil presents strong evidence that there is indeed a prevalence of bias in pain towards females. ${ }^{4}$ Although not all studies found a statistically significant difference between the sexes, when examining the overall trends present in the data, the results overwhelmingly indicate that females suffer more than men from chronic pain disorders (e.g., back pain, migraine, osteoarthritis, widespread pain, and musculoskeletal, oral, and neuropathic pain). Also, trends in basic pain sensitivity in healthy human subjects have shown that females experience increased sensitivity to electrical stimulation, ischemic pain, cold and heat pain, pressure pain, and muscle pain. These findings are in contrast with a review of 122 studies examining sex differences in laboratory pain sensitivity where the results showed no overall sex differences . ${ }^{6}$ Dr. Mogil points out, however, that, upon closer examination of the review, it can be argued that rather stringent inclusion criteria were used to indicate a 
significant difference between males and females. This is especially true when the sensitivity of multiple body parts was tested in a given study. For a sex difference to be considered significant, $50 \%$ or more of the areas tested had to show a sex difference. ${ }^{6}$ Dr. Mogil suggests that, if there truly was no difference between the sexes, the "absolute difference of male-female comparisons should be randomly distributed around zero". "When the results of the 122 studies are examined in this way, there is sufficient evidence to conclude that there is greater pain sensitivity in females and a need to examine sex differences in basic pain research. $^{4}$

The second barrier to studying sex differences in the basic mechanisms of pain has been the possible complication of variable hormone levels between the sexes. A commonly held view is that the hormones involved in the reproductive cycle of female mammals cause significant variability in behavioural results. In contrast, a recent study examined a large database of free- cycling female and male mice of varying genotypes to determine if results of tests using the tail-flick assay (using a $49^{\circ} \mathrm{C}$ stimulus) and the formalin model showed any variability between sexes. ${ }^{7}$ Study results showed no difference in variability between sexes for the tail-flick test, and it was actually males in the formalin test that showed the most variability in their responses. It was suggested that these results might be due to hormonal differences in males resulting from dominance hierarchies and fighting in their home cages, as defeat in these encounters has been shown to produce analgesia. ${ }^{7,8}$ These hormonal differences in male mice appear to be equal to, or perhaps greater than, those in females due to the estrus cycles. While these databases were examined for behaviours affecting only mice, it would appear that there is little cause to fear the estrus cycle in studies using animal models generally. In addition, it should be acknowledged that other areas of research use female subjects successfully on a regular basis without examining the estrus cycle. Studies using animal models of spinal cord injury and the experimental autoimmune encephalomyelitis (EAE) model used in multiple sclerosis research traditionally conduct research on female rodents (mice and rats) mainly due to husbandry concerns. Finally, there are also ways to control for the influence of hormones experimentally if they are considered a contributing factor to a specific research question. Researchers can eliminate the influence of sex hormones through physical or chemical castration in subgroups of their sample if they predict that sex hormones, not other factors, are driving the difference between groups. Taken together, it seems there is little reason to exclude female subjects from studies based solely on hormonal differences.
The third barrier to studying sex differences in basic pain research has been the observation that sex often interacts with other secondary factors. This can be seen with different genotypes in both rats and mice. ${ }^{9-11}$ Other factors shown to have an influence on both sex and pain include social interaction (compared with males, female mice interact more with their sick cage mates, which may be a form of analgesia), stress, genetics, and prenatal inflammation/stress. $^{4,7}$ It is surprising that a secondary factor, such as interaction, is considered a barrier to examining sex differences in pain when, in reality, the identification of more variables that influence pain helps gain a better understanding of how pain affects individuals. Pain is a complex phenomenon that is influenced by biological, psychological, and social factors, so it should not be surprising that there are secondary factors that interact with both pain and sex. For example, most clinical patients with chronic pain report depression and anxiety. Research has also shown that there is a higher prevalence of these disorders in females, so it should not be surprising that more females than males with chronic pain also experience depression and anxiety. Both acute and chronic pain will never be fully understood without considering possible secondary interacting factors in both basic and clinical research. Although this makes pain more challenging to understand from a mechanistic viewpoint, accounting for variability due to these factors and how they interact with sex will allow for a more complete understanding of pain and improve the development of analgesic therapies.

Despite these barriers, some researchers (both clinical and basic scientists) have embraced the study of sex differences. Results from these studies have revealed interesting and clinically important sex differences with regard to analgesics and the development of new therapeutic targets. For example, several underlying cellular differences in males and females can affect the effectiveness of morphine and sex steroid analgesia. Expression of $\mu$ and/or $\kappa$-opioid receptor heterodimers is regulated by spinal estrogen synthesis, which means that females often have a higher expression of these heterodimers. ${ }^{12}$ As this difference is controlled by estrogen synthesis, it may explain why morphine analgesia is dependent on the $\kappa$-opioid receptor gene in females but not in males. Another interesting biological difference between males and females is the apparent existence of two different neuronal circuits for stress-induced analgesia, $\kappa$-opioid analgesia, morphine tolerance, and morphine hyperalgesia. $^{13-16}$ It appears that the pathway in males is dependent on N-methyl-D-aspartate (NMDA)-type glutamate receptors, while the female pathway depends on melanocortin 1 receptors (MC1Rs). Results of studies examining these two 
pathways have shown that a non-competitive antagonist of the NMDA receptor will block analgesia in males but not in females at any dose, suggesting sex-specific pathways. ${ }^{14}$ Even more interestingly, a genetic lack of the MC1R in female mice will not result in a loss of analgesia (or morphine tolerance and hyperalgesia); instead, female mice can adopt a "male" phenotype and become responsive to NMDA receptor antagonists. This indicates that females can actually make use of the "male" pathway when their preferred MC1R pathway is blocked. ${ }^{16,17}$ $\mathrm{N}$-methyl-D-aspartate receptor antagonists are also effective in ovariectomized animals but can be rendered ineffective again with hormone therapy, showing that the MC1R pathway is hormone dependent. ${ }^{15,17}$ Research surrounding the MC1R pathway is also directly clinically applicable, as natural-born female redheads have a mutated form of the MC1R gene, making them more sensitive to certain types of analgesics (e.g., pentazocine) when compared with other women and all men. ${ }^{15}$

Previously explored sex differences have been centred on analgesia for acute pain; however, sex differences in chronic pain research have also been found. It is now well established that chronic pain develops and propagates through activation of both the immune system and glial cells in the spinal cord. Toll-like receptors (TLRs), particularly TLR4 (found on glial cells), are thought to be necessary to initiate the immune response needed for the development of chronic pain, since mice with dysfunctional TLR4 show reduced allodynia and hyperalgesia after nerve injury. ${ }^{18,19}$ Then again, in the mouse strain, $\mathrm{C} 3 \mathrm{H} /$ HeJ, with a mutation in the TLR4 gene, only male mice exhibit this reduced pain sensitivity. In addition, when testing female mice, the use of both agonists and antagonists to manipulate the TLR4 receptor resulted in no change in pain sensitivity, suggesting that chronic pain in females may be mediated by a different pathway. ${ }^{19}$ Importantly, if TRL4 were to be considered a therapeutic target for future drug development, it is possible that only males would benefit from these drugs. This is an important discovery not only for understanding possible mechanisms of chronic pain between the sexes but also for consideration for future drug development.

Current research showing differences in the mechanisms of pain/analgesia between the sexes along with the known clinical differences in both pain sensitivity and chronic pain prevalence reinforce the need for research into sex differences in pain. The discovery of potential sex differences in the development and propagation of chronic pain and the effectiveness of analgesics between the sexes will allow for a better overall understanding of both acute and chronic pain as well as the development of better drugs and therapies.

\section{Différences liées au sexe dans la douleur aiguë et chronique: une perspective du laboratoire au chevet du patient}

La douleur chronique affecte la vie quotidienne de millions de personnes. On estime que près d'un quart de la population mondiale souffre de douleur chronique, ce qui en fait le problème de santé humaine le plus répandu. ${ }^{1}$ Les estimations les plus récentes de prévalence de la douleur chronique au Canada indiquent qu'environ $20 \%$ de la population souffre de douleur chronique, et que près de $80 \%$ de ces personnes évaluent leur douleur comme étant de modérée à forte. ${ }^{2}$ Fait intéressant, on remarque une plus forte sensibilité à la douleur et une prévalence plus élevée de cas de douleur chronique chez les femmes que chez les hommes. ${ }^{3,4}$ À ce jour toutefois, la recherche fondamentale s'est peu intéressée à ces différences entre sexes d'un point de vue mécaniste, les chercheurs mettant en doute les données probantes ou les écartant tout simplement. Néanmoins, de nombreuses études et une revue récente de la littérature fournissent des données probantes convaincantes attestant qu'il existe en effet des différences entre les sexes en matière de douleur chronique et aiguë. ${ }^{4}$

Les différences entre les sexes ont été examinées dans de nombreux domaines de la recherche biologique, et tout particulièrement dans le domaine de la neuroscience moléculaire. Plusieurs études ont montré qu'il existe des différences entre les sexes d'un point de vue somatique et hormonal au niveau moléculaire, et que ces différences moléculaires se reflètent souvent en différences dans le pronostic et la progression d'une maladie donnée entre les sexes. La maladie d'Alzheimer, la dépression, la maladie de Huntington, l'ischémie cérébrale, et certains modèles de capacité cognitive en sont des exemples. ${ }^{5}$ Malgré les différences entre les sexes bien décrites dans la douleur et dans d'autres modèles pathologiques, la recherche fondamentale en douleur a été lente à adopter une étude méthodique de ces différences. Parmi les obstacles fréquemment rencontrés à l'étude des différences entre les sexes dans les mécanismes fondamentaux de la douleur, citons les doutes quant au bien-fondé d'étudier ces différences (le résultat d'études contradictoires dévaluant l'importance des études sur les sexes), les complications possibles liées à différentes influences hormonales sur le comportement, et la complexité d'essayer de déterminer les rôles enchevêtrés du sexe, de la douleur, et de facteurs secondaires associés à la douleur (par ex., la dépression). 
Un article de synthèse récent du Dr Jeffery Mogil de l'Université McGill a récemment traité de ces questions. En réexaminant des études cliniques et fondamentales portant sur les différences entre les sexes, le Dr Mogil a présenté des données probantes fiables soutenant qu'il existe véritablement un biais de prévalence de la douleur chez les femmes. ${ }^{4}$ Bien que les études examinées n'aient pas toutes observé de différence significative d'un point de vue statistique entre les sexes, lorsqu'on considère les tendances globales des données, les résultats indiquent sans équivoque que les femmes souffrent de troubles de douleur chronique tels que maux de dos, migraine, ostéoarthrite, douleur généralisée, douleurs musculosquelettiques, orales et neuropathiques plus que les hommes. En outre, les tendances de la sensibilité de base à la douleur chez les personnes en bonne santé ont démontré que les femmes possédaient une sensibilité accrue à la stimulation électrique, à la douleur ischémique, à la douleur liée au froid et à la chaleur, à la douleur de pression et à la douleur musculaire. Ces résultats sont contraires à ceux d'un article de synthèse examinant 122 études portant sur les différences entre les sexes en matière de sensibilité à la douleur en laboratoire, dans laquelle aucune différence globale entre les sexes n'avait été observée. ${ }^{6}$ Le Dr Mogil souligne toutefois qu'en examinant plus attentivement cet article, on peut prétendre que les critères d'inclusion pour qu'il y ait différence significative entre les hommes et les femmes étaient plutôt rigoureux. Ceci est particulièrement vrai lorsque la sensibilité de plusieurs parties du corps était testée dans une des études. Pour qu'une différence entre les sexes soit considérée comme étant significative, $50 \%$ ou plus des zones testées devaient montrer une différence entre les sexes. ${ }^{6}$ Le Dr Mogil suggère que, s'il n'y avait véritablement aucune différence entre les sexes, alors la «différence absolue des comparaisons homme-femme devrait se distribuer aléatoirement autour de zéro ». ${ }^{4}$ Lorsque les résultats des 122 études sont examinés de cette façon, les données probantes sont suffisantes pour conclure que les femmes manifestent une plus grande sensibilité à la douleur et qu'il faut étudier les différences entre les sexes dans la recherche fondamentale sur la douleur. ${ }^{4}$

Le deuxième obstacle à l'étude des différences entre les sexes dans les mécanismes fondamentaux de la douleur consiste en la complication possible que présentent des niveaux hormonaux variables entre les sexes. Une croyance fréquente voudrait que les hormones impliquées dans le cycle reproducteur des mammifères femelles provoquent une variabilité significative dans les résultats comportementaux. Un article récent a toutefois passé en revue une base de données imposante portant sur des souris mâles et des souris femelles ovulant normalement de génotypes divers, évaluées à l'aide d'un test de sensibilité à la pression (utilisant un stimulus à $49^{\circ} \mathrm{C}$ ) ainsi qu'un test à la formaline pour mesurer les différences de variabilité de leurs résultats. ${ }^{7}$ On a découvert qu'il n'y avait pas de différence de variabilité entre les sexes pour le test de sensibilité à la pression et que, dans le test à la formaline, les mâles étaient en fait ceux qui ont démontré la plus grande variabilité dans leurs réponses. Il a été suggéré que ces résultats pourraient être dus à des différences hormonales chez les mâles découlant de hiérarchies de dominance et de combats dans leurs cages d'origine, étant donné qu'il a été démontré qu'une défaite dans ces luttes provoquait une analgésie. ${ }^{7,8}$ Ces différences hormonales chez les souris mâles semblent être équivalentes, voire plus importantes, que celles observées chez les femelles à cause du cycle d'ovulation. Ces bases de données n'ont été examinées que pour évaluer les comportements affectant les souris; toutefois, il semble qu'il n'y ait pas de raison de craindre l'influence du cycle d'ovulation dans les études portant sur des modèles animaux en général. En outre, il convient de garder à l'esprit qu'il existe d'autres champs de recherche qui utilisent des sujets féminins avec succès et ce, sans examiner le cycle d'ovulation sur une base régulière. Les modèles animaux de lésions à la moelle épinière et le modèle d'EAE, utilisés en recherche sur la sclérose en plaque, sont traditionnellement des rongeurs femelles (souris et rats), principalement en raison de questions d'élevage. Enfin, il existe également des façons de contrôler de façon expérimentale l'influence des hormones si l'on pense qu'elles pourraient contribuer à une question de recherche spécifique. Les chercheurs peuvent éliminer l'influence des hormones sexuelles par castration physique ou chimique dans des sous-groupes de leurs échantillons s'ils anticipent que les hormones sexuelles, et non pas d'autres facteurs, seront à l'origine des différences entre les groupes. Si l'on tient compte de tous ces éléments, les arguments invoqués pour justifier l'exclusion des sujets féminins d'études uniquement en raison de différences hormonales semblent bien faibles.

Le troisième obstacle à l'étude des différences liées au sexe dans la recherche fondamentale en douleur se fonde sur l'observation que le sexe interagit souvent avec d'autres facteurs secondaires, comme on peut le voir avec différents génotypes chez les rats et les souris. ${ }^{9-11}$ Parmi les autres facteurs dont on a démontré une influence sur le sexe et la douleur, citons l'interaction sociale (les souris femelles interagissent avec leurs compagnons de cage malades davantage que les mâles, ce qui pourrait être une forme d'analgésie), le stress, la génétique, et l'inflammation / le stress prénatal. ${ }^{4,7}$ Il est surprenant que des facteurs secondaires confondants soient considérés comme un obstacle à l'étude des différences entre les sexes en douleur; en réalité, l'identification de variables supplémentaires influençant la douleur nous aide à acquérir une meilleure compréhension de la façon dont la douleur 
affecte une personne. La douleur est un phénomène complexe influencé par des facteurs biologiques, psychologiques et sociaux; dès lors, le fait qu'il existe des facteurs secondaires interagissant aussi bien avec la douleur qu'avec le sexe ne devrait pas nous surprendre. Par exemple, la plupart des patients vus en clinique et souffrant de douleur chronique rapportent de la dépression et de l'anxiété. La recherche a également démontré qu'il y a une plus forte prévalence de ces troubles chez les patients de sexe féminin - il ne devrait donc pas être surprenant qu'un nombre plus élevé de femmes que d'hommes souffrant de douleur chronique rapportent également de la dépression ou de l'anxiété. La douleur, qu'elle soit aiguë ou chronique, ne sera jamais totalement comprise si l'on ne tient pas compte de facteurs secondaires présentant une possible interaction, aussi bien dans la recherche fondamentale que clinique. Bien que ces facteurs rendent la douleur plus difficile à comprendre d'un point de vue mécaniste, le fait de prendre en compte la variabilité liée à ces facteurs et la façon dont ils interagissent avec le sexe nous permettra d'acquérir une compréhension plus globale de la douleur, et donc d'améliorer la mise au point de traitements d'analgésie.

Malgré ces obstacles, certains chercheurs, aussi bien cliniciens que fondamentaux, se sont penchés sur l'étude des différences entre les sexes. Les résultats de ces études ont révélé des différences entre les sexes à la fois intéressantes et importantes d'un point de vue clinique en ce qui touche aux analgésiques et à la mise au point de nouvelles cibles thérapeutiques. Par exemple, plusieurs différences cellulaires sous-jacentes entre les mâles et les femelles peuvent affecter l'efficacité de la morphine et de l'analgésie des stéroïdes sexuels. L'expression des hétérodimères des récepteurs opiacés $\mu$ et/ou $\kappa$ est modifiée par la synthèse rachidienne de l'œstrogène; en d'autres termes, les femelles présentent souvent une expression plus forte de ces hétérodimères. $^{12}$ Étant donné que cette différence est contrôlée par la synthèse de l'œstrogène, cela pourrait expliquer pourquoi l'analgésie à la morphine dépend du gène du récepteur opiacé $\kappa$ chez les femelles mais pas chez les mâles. Une autre différence biologique intéressante réside dans l'existence apparente de deux circuits neuronaux différents chez les mâles et les femelles pour l'analgésie induite par le stress, l'analgésie par le récepteur opiacé $\kappa$, la tolérance à la morphine et l'hyperalgésie à la morphine. ${ }^{13-16}$ Il semble que la voie de transmission, chez les mâles, soit dépendante des récepteurs du glutamate de type N-méthyl-D-aspartate (NMDA), alors que la voie de transmission chez les femelles dépendrait des récepteurs à la mélanocortine de type 1 (MC1R). Des études portant sur ces deux voies de transmission ont révélé que, indépendamment de la dose, un antagoniste non compétitif du récepteur NMDA bloque l'analgésie chez les mâles mais pas chez les femelles, ce qui suggère l'existence de voies de transmission spécifiques au sexe. ${ }^{14}$ Fait encore plus intéressant, l'absence génétique du MC1R chez des souris femelles n'entraîne pas de perte d'analgésie (ou de tolérance à la morphine ou d'hyperalgésie); plutôt, les souris femelles peuvent adopter un phénotype 'mâle' et, par conséquent, répondre aux antagonistes des récepteurs NMDA. Ceci indique que les femelles peuvent en fait se servir de la voie de transmission 'mâle' lorsque leur voie de transmission privilégiée, le MC1R, est bloquée. ${ }^{16,17}$ Les antagonistes des récepteurs du N-méthyl-D-aspartate sont également efficaces chez les animaux ayant subi une ovariectomie, mais peuvent à nouveau être rendus inefficaces avec un traitement hormonal - ce qui démontre que la voie de transmission du MC1R est hormono-dépendante. ${ }^{15,17}$ La recherche entourant la voie de transmission du MC1R est également applicable directement en clinique, étant donné que les femmes rousses naturelles possèdent une forme mutée du gène $\mathrm{MC} 1 \mathrm{R}$, ce qui les rend plus sensibles à certains types d'analgésiques (par ex. la pentazocine) que d'autres femmes et tous les hommes. ${ }^{15}$

Les différences entre les sexes explorées par le passé se sont concentrées sur l'analgésie pour la douleur aiguë; toutefois, on a également trouvé des différences entre les sexes en recherche sur la douleur chronique. Il est désormais bien établi que la douleur chronique se développe et se propage par l'activation du système immunitaire et des cellules gliales de la moelle épinière. On pense que les récepteurs de type Toll (TLR), particulièrement le TLR4 (qu'on trouve sur les cellules gliales) sont nécessaires pour initier la réponse immunitaire nécessaire au développement de la douleur chronique; en effet, les souris possédant un TLR4 dysfonctionnel montrent une allodynie et une hyperalgésie réduites après une lésion nerveuse. ${ }^{18,19}$ Toutefois, chez la souche de souris $\mathrm{C} 3 \mathrm{H} / \mathrm{HeJ}$ présentant une mutation du gène TLR4, seules les souris mâles ont démontré cette sensibilité réduite à la douleur. En outre, dans des études portant sur les souris femelles, l'utilisation d'agonistes et d'antagonistes pour manipuler le récepteur TLR4 n'a pas eu d'effet sur la sensibilité à la douleur, ce qui suggère que la douleur chronique pourrait être médiée par une voie de transmission différente chez les femelles. ${ }^{19}$ Il est important de souligner que, si le TLR4 était envisagé comme cible thérapeutique pour la mise au point de médicaments à l'avenir, il est possible que seuls les mâles tireraient profit de ces médicaments. Il s'agit là d'une découverte importante, non seulement pour la compréhension des mécanismes possibles de la douleur chronique entre les sexes, mais également pour la mise au point future de médicaments.

Les recherches actuelles démontrant des différences dans les mécanismes de la douleur et de l'analgésie entre les sexes, en plus des différences cliniques connues au niveau de la sensibilité à la douleur et de la prévalence de la douleur chronique, renforcent le besoin de recherches 
portant sur les différences entre les sexes en matière de douleur. La découverte de différences potentielles entre les sexes au niveau du développement et de la propagation de la douleur chronique, ainsi que d'une efficacité différente des analgésiques entre les sexes, nous donnera une meilleure compréhension globale de la douleur aiguë et chronique et nous permettra de mettre au point de meilleurs médicaments et de meilleurs traitements.

Competing interests None declared.

\section{References}

1. Moulin DE, Clark AJ, Speechley M, Morley-Forster PK. Chronic pain in Canada: prevalence, treatment, impact, and the role of opioid analgesia. Pain Res Manag 2001; 7: 179-84.

2. Institute of Medicine. Relieving Pain in America: a Blueprint for Transforming Prevention, Care, Education, and Research. Washington, DC: National Academies Press; 2011: 1-382.

3. Ruau D, Liu LY, Clark JD, Angst MS, Butte AJ. Sex differences in reported pain cross 11,000 patients captured in electronic medical records. J Pain 2012; 13: 228-34.

4. Mogil JS. Sex differences in pain and pain inhibition: multiple explanations of a controversial phenomenon. Nat Rev Neurosci 2012; 13: 859-66.

5. Jazin E, Cahill L. Sex differences in molecular neuroscience: from fruit flies to humans. Nat Rev Neurosci 2010; 11: 9-17.

6. Racine M, Tousignant-Laflamme Y, Kloda LA, Dion D, Dupuis G, Choiniere $M$. A systematic literature review of 10 years of research on sex/gender and experimental pain perception - part 1: are there really differences between women and men? Pain 2012; 153: 602-18.

7. Mogil JS, Chanda ML. The case for the inclusion of female subjects in basic science studies of pain. Pain 2005; 117: 1-5.

8. Miczek KA, Thompson ML, Shuster L. Opioid-like analgesia in defeated mice. Science 1982; 215: 1520-2.
9. DeLeo JA, Rutkowski MD. Gender differences in rat neuropathic pain sensitivity is dependent on strain. Neurosci Lett 2000; 282: 197-9.

10. Lee DH, Chung K, Chung JM. Strain differences in adrenergic sensitivity of neuropathic pain behaviors in an experimental rat model. Neuroreport 1997; 8: 3453-6.

11. Mogil JS, Smith SB, O'Reilly MK, Plourde G. Influence of nociception and stress-induced antinociception on genetic variation in isoflurane anesthetic potency among mouse strains. Anesthesiology 2005; 103: 751-8.

12. Chakrabarti S, Liu NJ, Gintzler AR. Formation of $\mu$-/к-opioid receptor heterodimer is sex-dependent and mediates femalespecific opioid analgesia. Proc Natl Acad Sci USA 2010; 107: 20115-9.

13. Kavaliers $M$, Choleris E. Sex differences in N-methyl-D-aspartate involvement in $\kappa$-opioid and non-opioid predator-induced analgesia in mice. Brain Res 1997; 768: 30-6.

14. Bryant CD, Eitan $S$, Sinchak $K$, Fanselow MS, Evans CJ. NMDA receptor antagonism disrupts the development of morphine analgesic tolerance in male, but not female C57BL/6 J mice. Am J Physiol Regul Integ Comp Physiol 2006; 291: R315-26.

15. Mogil JS, Wilson SG, Chesler EJ, et al. The melanocortin-1 receptor gene mediates female-specific mechanisms of analgesia in mice and humans. Proc Natl Acad Sci USA 2003; 100: 4867-72.

16. Juni A, Cai $M$, Stankova $M$, et al. Sex-specific mediation of opioid-induced hyperalgesia by the malanocortin-1 receptor. Anesthesiology 2010; 112: 181-8.

17. Juni A, Klein $G$, Kowalczyk B, Ragnauth A, Kest B. Sex differences in hyperalgesia during morphine infusion: effect of gonadectomy and estrogen treatment. Neuropharmacology 2008; 54: 1264-70.

18. Tanga FY, Nutile-McMenemy N, DeLeo JA. The CNS role of Toll-like receptor 4 in innate neuroimmunity and painful neuropathy. Proc Natl Acad Sci USA 2005; 102: 5856-61.

19. Bettoni I, Comelli F, Rossini C, et al. Glial TLR4 receptor as new target to treat neuropathic pain: efficacy of a new receptor antagonist in a model of peripheral nerve injury in mice. Glia 2008; 56: 1312-9. 